\title{
Random-roughness hydrodynamic boundary conditions
}

\author{
Christian Kunert, ${ }^{1}$ Jens Harting, ${ }^{2,1}$ and Olga I. Vinogradova ${ }^{3,4}$ \\ ${ }^{1}$ Institute for Computational Physics, University of Stuttgart, Pfaffenwaldring 27, 70569 Stuttgart, Germany \\ ${ }^{2}$ Department of Applied Physics, Eindhoven University of Technology, \\ P.O. Box 513, 5600 MB Eindhoven, The Netherlands \\ ${ }^{3}$ A.N. Frumkin Institute of Physical Chemistry and Electrochemistry, \\ Russian Academy of Sciences, 31 Leninsky Prospect, 119991 Moscow, Russia \\ ${ }^{4}$ ITMC and DWI, RWTH Aachen, Pauwelsstr. 8, 52056 Aachen, Germany
}

(Dated: October 26, 2018)

\begin{abstract}
We report results of lattice Boltzmann simulations of a high-speed drainage of liquid films squeezed between a smooth sphere and a randomly rough plane. A significant decrease in the hydrodynamic resistance force as compared with that predicted for two smooth surfaces is observed. However, this force reduction does not represent slippage. The computed force is exactly the same as that between equivalent smooth surfaces obeying no-slip boundary conditions, but located at an intermediate position between peaks and valleys of asperities. The shift in hydrodynamic thickness is shown to depend on the height and density of roughness elements. Our results do not support some previous experimental conclusions on very large and shear-dependent boundary slip for similar systems.
\end{abstract}

PACS numbers: 83.50.Rp,68.08.-p

Introduction.- It has been recently well recognized that the famous no-slip boundary condition, for more than a hundred years applied to model experiments in fluid mechanics, reflected mostly a macroscopic character and insensitivity of old style experiments. Modern experiments concluded that although the no-slip postulate is valid for molecularly smooth hydrophilic surfaces down to contact [1 3], for many other systems it does not apply when the size of a system is reduced to microand nano scales. The changes in hydrodynamic behavior are caused by an impact of interfacial phenomena, first of all hydrophobicity and roughness, on the flow. The effect of hydrophobicity on the flow past smooth surfaces is reasonably clear and suggests an amount of slippage described by the condition $v_{s}=b \partial v / \partial z$ where $v_{s}$ is the slip velocity at the wall, $b$ the slip length, and the axis $z$ is normal to the surface. The assumption is justified theoretically 4 7 and was confirmed by surface force apparatus (SFA) 2], atomic force microscope (AFM) [1], and fluorescence cross-correlation (FCS) [3] experiments. Despite some remaining controversies in the data and amount of slip (cf. [8]), a concept of hydrophobic slippage is now widely accepted. If a liquid flows past a rough hydrophobic (i.e. superhydrophobic) surface, roughness may favor the formation of trapped gas bubbles, resulting in a large slip length 9 -14. For rough hydrophilic surfaces the situation is much less clear, and opposite experimental conclusions have been made: one is that roughness generates extremely large slip [15], and one is that it decreases the degree of slippage 16, 17]. More recent experimental data suggests that the description of flow near rough surfaces has to be corrected, but for a separation, not slip [18]. The theoretical description of such a flow represents a difficult, nearly insurmountable, problem. It has been solved only approximately, and only for a case of the periodic roughness and far-field flow with a conclusion that it may be possible to approximate the actual surface by a smooth one with the apparent slip boundary condition 1921 .

In this letter we address the fundamental, but still open questions (i) whether the effect of random roughness on the flow may be represented by replacing the no-slip condition on the exact boundary by an effective condition on the equivalent smooth surface, (ii) where this smooth surface is located, depending on geometric parameters of roughness, and (iii) does this effective condition represent that of slip or no-slip? We will quite generally assume that the flow near and far from the interface is a stable, laminar flow field.

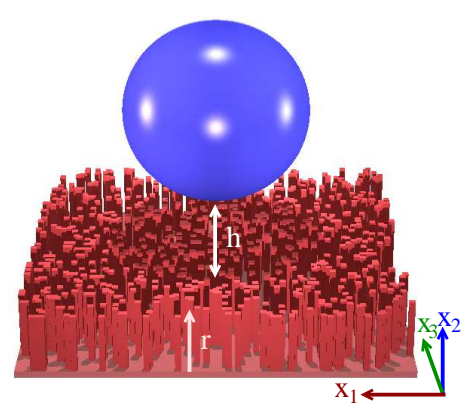

FIG. 1: (Color online) Sketch of the system: a sphere of radius $R$ approaches a rough surface with a fixed area fraction $\phi$ covered by roughness elements. The separation $h$ is defined on top of the surface roughness at position $x_{2}=r$.

General idea and models.- To address these issues we analyze the hydrodynamic interaction between a smooth sphere of radius $R$ and a rough plane (see Fig. (1). Beside its significance as a geometry of SFA/AFM dynamic force experiments, this allows us to explore both far and near-field flows in a single "experiment". As an 
initial application we study roughness elements of a fixed height $r$ that are distributed at random uncorrelated positions with a given probability $\phi$. Such a surface mimics a situation explored in recent experiments 15 17]. In Cartesian coordinates $\mathbf{x}=\left(x_{1}, x_{2}, x_{3}\right)$, a separation $h$ is defined on top of the roughness, $x_{2}=r$, which finds its definition in the AFM experiment 15, 18].

The exact solution, valid for an arbitrary separation, for a sphere approaching a smooth plane is given by theoretical solutions of Brenner and Maude [22, 23],

$$
\begin{gathered}
\frac{F_{1}}{F_{S t}}=-\frac{1}{3} \sinh \xi \\
\times\left(\sum_{n=1}^{\infty} \frac{n(n+1)\left[8 e^{(2 n+1) \xi}+2(2 n+3)(2 n-1)\right]}{(2 n-1)(2 n+3)\left[4 \sinh ^{2}\left(n+\frac{1}{2}\right) \xi-(2 n+1)^{2} \sinh ^{2} \xi\right]}\right. \\
\left.-\sum_{n=1}^{\infty} \frac{n(n+1)\left[(2 n+1)(2 n-1) e^{2 \xi}-(2 n+1)(2 n+3) e^{-2 \xi}\right]}{(2 n-1)(2 n+3)\left[4 \sinh ^{2}\left(n+\frac{1}{2}\right) \xi-(2 n+1)^{2} \sinh ^{2} \xi\right]}\right),
\end{gathered}
$$

with $F_{S t}=6 \pi \mu R v$, where $\mu$ is the dynamic viscosity, $v$ is the velocity, and $\cosh \xi=h / R, \xi<0$. The leading term of this expression can be evaluated as

$$
\frac{F_{2}}{F_{S t}} \sim 1+\frac{9}{8} \frac{R}{h}
$$

At large separations, $h \gg R$, the hydrodynamic force on a sphere turns to the Stokes formula, but at small distances, $h \ll R$, the drag force is inversely proportional to the gap, $F_{2} / F_{S t} \rightarrow 9 R /(8 h)$. A consequence of this lubrication effect is that the sphere would never touch the wall in a finite time. The flow in the vicinity of a rough surface should deviate from these predictions. A possible assumption is that the boundary condition at the plane $x_{2}=r$ should be written as a slip condition [15]. To investigate this scenario we suggest to present a force as a product of Eq. 2 and a correction for slip

$$
\frac{F_{3}}{F_{S t}} \sim\left(1+\frac{9}{8} \frac{R}{h}\right) f^{*}
$$

where this correction, $f^{*}$, is taken to be equal as predicted for a lubrication force between a no-slip surface and a surface with partial slip [24].

$$
f^{*}=\frac{1}{4}\left(1+\frac{3 h}{2 b}\left[\left(1+\frac{h}{4 b}\right) \ln \left(1+\frac{4 b}{h}\right)-1\right]\right) .
$$

Another assumption would be that the rough surface is hydrodynamically equivalent to a smooth one located somewhere between the top and bottom of rugosities (at $x_{2}=r_{\text {eff }}=r-s$ ). As found in [18]), the force can be represented as

$$
\frac{F_{4}}{F_{S t}} \sim 1+\frac{9}{8} \frac{R}{h+s}
$$

At small $h$ expressions 3 and 4 give different asymptotic behavior of a drag force, $F_{3} / F_{S t} \rightarrow 9 R /(32 h)$, and $F_{4} / F_{S t} \rightarrow 9 R /(8 s)$. While the second scenario allows a sphere to touch a plane, in the first model this is impossible since the drag force diverges (but differs from the standard lubrication asymptotics by a factor of 4 ). Thus, a drainage study allows to distinguish between these two models of hydrodynamic flow past rough surfaces.

Simulation method.- We apply the lattice Boltzmann (LB) method to simulate the flow field between a smooth sphere approaching a rough plane [25 27]. The method allows precise measurements of the force acting on the sphere and to explore the very large range of parameters. Besides that, in our simulations we can consider a "clean" situation of a hydrodynamic force and avoid effects of surface forces which significantly complicate the analysis of SFA/AFM data. Since the method is well established, we only shortly describe it here. By using a discretized and linearized version of Boltzmann's equation

$$
n_{i}\left(\mathbf{x}+\mathbf{c}_{i}, t+1\right)-n_{i}(\mathbf{x}, t)=\sum_{j} \Lambda_{i j}\left(n_{j}^{\mathrm{eq}}-n_{j}(\mathbf{x}, t)\right),
$$

the LB approach allows to fully resolve the hydrodynamics 28]. Positions $\mathbf{x}$ are discretized on a 3D lattice with 19 discrete velocities $\mathbf{c}_{i}$ pointing to neighboring sites. Each $\mathbf{c}_{i}$ relates to a single particle distribution function $n_{i}(\mathbf{x}, t)$ which is advected to neighboring sites at every time step. Then, $n_{i}(\mathbf{x}, t)$ is relaxed towards a local equilibrium $n_{i}^{\text {eq }}(\rho, \mathbf{j})$ with a rate given by the matrix elements $\Lambda_{i j}$. Mass $\rho$ and momentum $\mathbf{j}$ as given by moments of $n_{i}(\mathbf{x}, t)$ are conserved. We use the natural units of the system, i.e. the lattice constant $\delta \mathrm{x}$ for the length and the time step $\delta$ t for time. Massive particles are described by a continuously moving boundary which is discretized on the lattice. Momentum from the particle to the fluid is transfered such that the fluid velocity at the boundary equals the particle's surface velocity. Since the momentum transferred from the fluid to the particle is known, the hydrodynamic force can be recorded. If not stated otherwise, the $256^{3} \delta \mathrm{x}^{3}$ system contains a sphere with radius $R=16 \delta \mathrm{x}$ which is moved in $y$ direction at constant velocity $v=10^{-3} \delta \mathrm{x} / \delta \mathrm{t}$. The fluid density is kept constant and the kinematic viscosity is $\mu / \rho=0.1$ resulting in a Reynolds number $R e=0.16$. No-slip surfaces are described by mid-grid bounce back boundaries and a slip boundary is implemented by a repulsive mean-field force acting between fluid and surface [7, 12]. We carefully checked the influence of system size, radius, and separation to insure that finite size and resolution effects are negligible [29]. Also, by testing different resolutions we assured that a lateral width of roughness elements of $1 \delta \mathrm{x}$ is sufficient.

Results and discussion.- We test our method by measuring the hydrodynamic interaction between smooth surfaces. Fig. 2 shows the normalized hydrodynamic force for two simulation sets. In the first one, a sphere of $R=8 \delta \mathrm{x}$ is driven with $v=10^{-4} \delta \mathrm{x} / \delta \mathrm{t}$. In the second run the sphere is twice as large, $R=16 \delta \mathrm{x}$, 


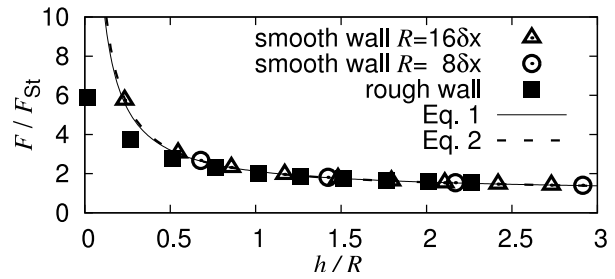

FIG. 2: Hydrodynamic force acting on a sphere with radius $R=16 \delta$ x (triangles) and $R=8 \delta \mathrm{x}$ (circles) driven to a smooth wall with velocity $v=10^{-3} \delta \mathrm{x} / \delta \mathrm{t}$ and $v=10^{-4} \delta \mathrm{x} / \delta \mathrm{t}$, correspondingly. The solid and dashed curves are calculations of the force expected with no-slip boundary conditions at the wall (Eqs. 1 2). Squares are the results measured for a rough wall $(\phi=4 \%, r=10 \delta \mathrm{x})$.

and the driving velocity is an order of magnitude larger, $v=10^{-3} \delta \mathrm{x} / \delta$ t. Fig. 2 includes the exact theoretical curve, Eq. 1 The fit is excellent for all separations, indicating that large shear rates do not induce any slip, a conclusion which does not support recent experimental data [8]. Note that the first-order approximation, Eq. 2] practically coincides with the exact solution. These simulations demonstrate that finite size effects and resolution effects can be well controlled: for $h<2 R$ a $256^{3} \delta \mathrm{x}^{3}$ system is found to be sufficient to avoid artefacts at large separations $h$ [29]. Separations $<1 \delta \mathrm{x}$ are excluded from the analysis since the finite resolution leads to larger deviations. Also included in Fig. 2 is a normalized force measured near a rough wall $(\phi=4 \%, r=10 \delta \mathrm{x})$, which at small distances is much smaller than predicted by Eq. [1. This is qualitatively consistent with the AFM observations [1, 15], but in contrast to the SFA data [17], which likely reflects a different way of a definition of zero separation in the SFA (at the bottom of asperities).

To examine the significance of roughness more closely, the force curves from Fig. 2 are reproduced in Fig. 3 in different coordinates. Figs. $3 \mathrm{a}$ and $\mathrm{b}$ are intended to indicate that both near field and far field theoretical asymptotics for smooth surfaces are well reproduced in simulations. Figs. 3r and d show that simulation data for a rough surface $(\phi=4 \%, r=10 \delta \mathrm{x})$ show deviations from the behavior predicted by Eq. 1. A possible explanation for this discrepancy is that we invoke slippage at the wall, as modeled by Eq. 3. This is illustrated in Figs. 3r and d, where the simulation data are compared with another theoretical calculation in which a constant slip length of $b=2.55 \delta \mathrm{x}$, obtained from the best possible fit of the force curve, is incorporated in the model. This has the effect of decreasing the force, and it provides a reasonable fit to the data down to $h / R \sim 3$, but at smaller gap the simple model of slip fails to describe simulation data, by predicting a larger force and its different asymptotic behavior. This suggests, that it can only be considered as a first approximation, valid at large distances from the wall. This conclusion is con-
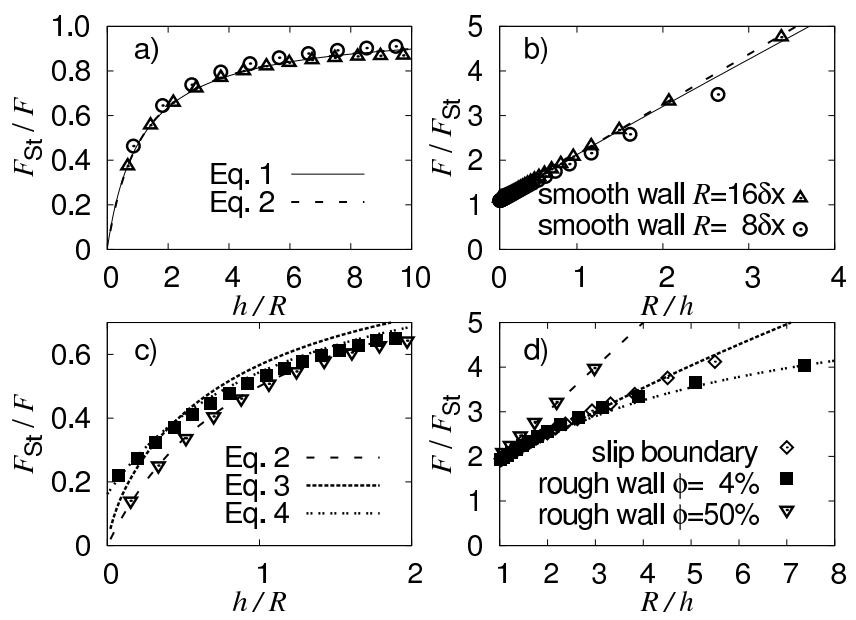

FIG. 3: Hydrodynamic force plotted in different coordinates. $(\mathrm{a}, \mathrm{b})$ the data sets for smooth surfaces reproduced from Fig 2 (c,d) show the force for two rough planes with $r=10 \delta \mathrm{x}$, $\phi=4 \%$ and $\phi=50 \%$. For $\phi=4 \%$ the asymptotic behavior for small $h$ cannot be fitted with a slip, but the assumption of an effective boundary position holds. The values for $\phi=50 \%$ recover the case of a flat surface at $r$. The data for a smooth slip boundary confirms the validity of Eq. 3

sistent with early results obtained for a far field situation 19 21], but does not support recent AFM data [15]. However, as shown by the simulation data, Eq. 3 is well applicable in the case of a slippery wall. An alternative explanation for the smaller force compared to the theory for smooth surfaces, Eq. 1] can be obtained if we assume that the location of an equivalent effective wall, where no-slip boundary conditions are applied, should be shifted, as modeled by Eq. 4. A corresponding theoretical calculation of the drag force is shown in Figs. $3 \mathrm{r}$ and d. This estimate requires knowledge of the effective wall position $r_{\text {eff }}$. The value $r_{\text {eff }}=7.86 \delta \mathrm{x}$ was obtained from the fit of the measured force curve and is enough to give a good match to the data at very small distances, which confirms the conclusions of a recent experiment [18].
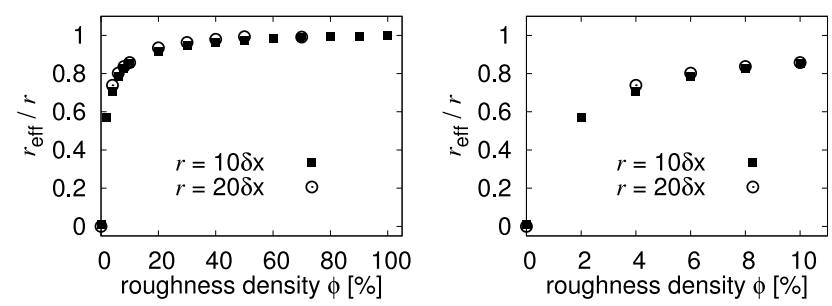

FIG. 4: Effective height $r_{\text {eff }}$ normalized by the maximum height $r$ as a function of a density of roughness elements, $\phi$, for $r=10 \delta \mathrm{x}$ and $r=20 \delta \mathrm{x}$ plotted in different scales.

By performing similar fits for a variety of drainage runs with different $\phi$ and for surfaces with different height of roughness elements $(r=10 \delta \mathrm{x}$ and $r=20 \delta \mathrm{x})$ as well as its different lateral width ( $\delta \mathrm{x}$ and $2 \delta \mathrm{x})$ we find that the 
same conclusion is valid for all situations, but $r_{\text {eff }} / r$ is itself a function of $\phi$ (being surprisingly insensitive to the value of $r$ ). In Fig. 4 we examine this in more detail. The simulation data show that $r_{\text {eff }}$ required to fit each run increases from 0 to $r$ very rapidly, so that at $\phi=20 \%$ it is already above $0.9 r$, and at $\phi=50 \%$ it is almost equal to $r$. This is illustrated by including the data obtained for a larger density of roughness elements $(\phi=50 \%, r=10 \delta \mathrm{x})$ in Fig. 3r, that do not show a discernible deviation from the theoretical predictions for smooth surfaces. Thus, a small number of roughness elements has enormous influence on film drainage, confirming earlier theoretical ideas [30].

Conclusion.- We have presented lattice Boltzmann simulations describing the drainage of a liquid confined between a smooth sphere and a randomly rough plate. The measured force is smaller than predicted for two smooth surfaces if the standard no-slip boundary conditions are used in the calculation. What our results show, however, is that at small separations the force is even weaker and shows different asymptotics than expected if we invoke slippage at the smooth fluid-solid interfaces. To explain this we use the model of a no-slip wall, located at an intermediate position (controlled by the density of roughness elements) between top and bottom of asperities. Calculations based on this model provide an excellent description of the simulation data. Besides this, by proving a correctness of this simple model to describe flow past a randomly rough surface, we have suggested a validity of a number of simple formulas for a hydrodynamic drag force. Although formally they can only be considered as first-order approximations, their accuracy is confirmed by simulation. Our results open the possibility of solving quantitatively many fundamental hydrodynamic problems involving randomly-rough interfaces, including contact angle dynamics, coagulation and more.

We acknowledge A.J.C. Ladd for his hospitality (C. Kunert) and access to his simulation code, the DFG for financial support (grants Vi 243/1-3 and Ha 4382/2$1)$, and SSC Karlsruhe for computing time.

[1] O. I. Vinogradova and G. E. Yakubov. Langmuir, 19:1227, 2003.

[2] C. Cottin-Bizonne, B. Cross, A. Steinberger, and E. Charlaix. Phys. Rev. Lett., 94:056102, 2005.

[3] O. I. Vinogradova, K. Koynov, A. Best, and F. Feuillebois. Phys. Rev. Lett., 102:118302, 2009.
[4] O. I. Vinogradova. Int. J. Miner. Process., 56:31 - 60, 1999.

[5] J. L. Barrat and L. Bocquet. Phys. Rev. Lett., 82:4671 4674, 1999.

[6] D. Andrienko, B. Dünweg, and O. I. Vinogradova. J. Chem. Phys., 119:13106, 2003.

[7] J. Harting, C. Kunert, and H. Herrmann. Europhys. Lett., 75:328, 2006.

[8] E. Lauga, M. P. Brenner, and H. A. Stone. In C. Tropea, A. Yarin, and J. F. Foss, editors, Handbook of Experimental Fluid Dynamics, chapter 19, pp 1219-1240. Springer, NY, 2007.

[9] P. Joseph, C. Cottin-Bizonne, J. M. Benoi, C. Ybert, C. Journet, P. Tabeling, and L. Bocquet. Phys. Rev. Lett., 97:156104, 2006.

[10] J. Ou and J. P. Rothstein. Physics of Fluids, 17:103606, October 2005.

[11] F. Feuillebois, M. Z. Bazant, and O. I. Vinogradova. Phys. Rev. Lett., 102:026001, 2009.

[12] J. Hyväluoma and J. Harting. Phys. Rev. Lett., 100:246001, 2008.

[13] M. Sbragaglia, R. Benzi, L. Biferale, S. Succi, and F. Toschi. Phys. Rev. Lett., 97:204503, 2006.

[14] H. Kusumaatmaja, M. L. Blow, A. Dupuis, and J. M.Yeomans. Europhys. Lett., 91:36003, 2008.

[15] E. Bonaccurso, H.-J. Butt, and V. S. J. Craig. Phys. Rev. Lett., 90:144501, 2003.

[16] S. Granick, Y. Zhu, and H. Lee. Nat. Mater., 2:221 227, 2003.

[17] Y. X. Zhu and S. Granick. Phys. Rev. Lett., 88:106102, 2002.

[18] O. I. Vinogradova and G. E. Yakubov. Phys. Rev. E, 73:045302(R), 2006.

[19] K. Sarkar and A. Prosperetti. J. Fluid Mech., 316:223, 1996.

[20] N. Lecoq, R. Anthore, B. Cichocki, P. Szymczak, and F. Feuillebois. J. Fluid Mech., 513:247, 2004.

[21] C. Kunert and J. Harting. Phys. Rev. Lett, 99:176001, 2007.

[22] H. Brenner. Chem. Eng. Sci., 16:242-251, 1961.

[23] A. D. Maude. British J. Appl. Phys., 12:293-295, 1961.

[24] O. I. Vinogradova. Langmuir, 11:2213 - 2220, 1995.

[25] A. J. C. Ladd and R. Verberg. J. Stat. Phys., 104:1191, 2001.

[26] D. d'Humières, I. Ginzburg, M. Krafczyk, P. Lallemand, and L.-S. Luo. Phil. Trans. R. Soc. Lond. A, 360:437, 2002.

[27] F. J. Higuera, S. Succi, and R. Benzi. Europhys. Lett., 9:345, 1989.

[28] S. Succi. The lattice Boltzmann equation for fluid dynamics and beyond. Oxford University Press, 2001.

[29] C. Kunert and J. Harting. Proceedings of MNF, 2009.

[30] T. H. Fan and O. I. Vinogradova. Phys. Rev. E, 72:066306, 2005. 